\title{
Simulations of gas flow in gas atomisation of liquid metals and validation experiments
}

\author{
Christopher Hulme-Smith*1, Arun Kamalasekaran ${ }^{1}$, Stefan Sundin² \\ ${ }^{1}$ Department of Materials Science and Engineering, KTH Royal Institute of Technology, \\ Stockholm, Sweden \\ ${ }^{2}$ Powder Expertise Advanced Research Laboratory (PEARL), Erasteel Kloster AB, \\ Söderfors, Sweden \\ *Corresponding author email: chrihs@kth.se
}

\begin{abstract}
The production of metal powder by gas atomisation generates feedstock for many manufacturing techniques, including hot isostatic pressing, laser cladding and, of current interest, powder bed additive manufacturing. While many thousands of alloys are commercially available in bulk form, fewer than fifty are widely available as powders suitable for additive manufacturing. This is due to difficulties in controlling droplet size distribution and avoiding particle defects. Indeed, a typical gas atomisation process that attempts to make powder for powder bed additive manufacturing achieves a yield of suitable powder below $50 \%$. Several studies have simulated the gas atomisation process or part of it, but few validate the models directly. The current work aims to take a first step towards making a holistic and fully validated model for gas atomisation. The gas flow from de Laval nozzles under conditions similar to those used in gas atomisation has been simulated using computational fluid dynamics and experiments to validate these simulations have been performed using shadowgraphy. A validated model can be used as part of a larger model to predict the phenomena that occur in gas atomisation and thereby refine the process to improve the production yield of powders, especially for additive manufacturing.
\end{abstract}

\section{Keywords}

Gas atomisation, powder metallurgy, computational fluid dynamics, shadowgraphy.

\section{Introduction}

Metal powders are made in a variety of ways, depending on the ultimate application. Techniques such as ball milling [1-3], jet milling, electrolytic deposition, metal salt reduction, chemical reactions have all been demonstrated and, in some cases, commercialised [4]. However, the vast majority of metal powders made on a commercial scale are atomised. Within atomisation there are distinct techniques including impact atomisation, centrifugal atomisation, ultrasonic atomisation, water atomisation and gas atomisation [5]. One can also include rotating electrode processes, using both plasma and electric arcs as atomising processes as metal is melted at the end of a rapidly spinning electrode and thrown outwards as droplets [5]. Of these, it is generally accepted that the most productive technique is water atomisation. However, the majority of current research interest surrounds gas atomisation. This is because it can generate particles of high sphericity in the size range that is of interest to many processes, including powder bed additive manufacturing, which is a group of processes that may be referred to as 3D printing.

In powder bed additive manufacturing of metals, thin layers of metal powder are spread and selectively melted to build up a three dimensional component. The thickness of these layers depends on the individual printer being used and the technology used to join the powder 
together into a dense component. If a laser is used as a heat source, layers are typically 30 $50 \mu \mathrm{m}$ thick but can be up to $100 \mu \mathrm{m}$ [3,6-8]. A similar thickness is used if powder is joined using a binder (binder jetting) [9]. However, if an electron beam is used instead, metal powder absorbs heat more efficiently and so thicker layers are possible. Layers are typically 50$100 \mu \mathrm{m}$ thick, but can be as high as $150 \mu \mathrm{m}$ [6]. This affects the particle size range that is considered acceptable, with larger particles specified for electron beam melting processes. Typically, laser powder bed fusion demands powders $\sim 35 \mu \mathrm{m}$ in diameter, while electron beam powder bed fusion requires particles $\sim 77 \mu \mathrm{m}$ in diameter [10].

The demands on feedstock for powder bed additive manufacturing include restrictions on particle size, particle shape, chemical purity and powder flow and spreading behaviour [11]. All powder particles must be smaller than the individual layers in the process, but a large abundance of very fine particles can lead to agglomeration and difficulties in powder handling. Irregular particles are likely to interlock and inhibit powder flow and spreading. Fine particles are also likely to reduce powder flow and spreading [7]. Insufficient spreading will result in defects within powder layers, which are very likely to be inherited by the final component and can even cause the print run to fail. Gas atomisation is the powder production technique that is able to produce suitable powder in the most economical way. Other techniques such as centrifugal atomisation and the plasma rotating electrode process are also able to meet these demands and even produce powder with higher sphericity and a narrower particle size distribution, but at a significantly higher cost [5].

Atomising of liquid metals presents a unique set of challenges, compared to other atomising processes, as the liquid that is to be atomised exhibits a very high density, a high (dynamic) viscosity and very high surface [4]. This makes breakup very difficult and extreme conditions are required to achieve it, with supersonic gas jets and high-pressure water jets required to achieve the desired droplet size. In addition, the high temperatures presents many technical and safety challenges that are beyond the scope of this study but are common in the context of metals processing. Gas atomisation is attractive because of the large number of parameters that may be used to control the size of the powder particles that are produced by the atomising process. Such parameters include the geometry of the gas jets, the temperature and pressure of the gas, the temperature of the molten metal and the ratio to the flow rate of the metal and the gas [5].

Nevertheless, gas atomisation is far from optimised for metal powder production. The size distribution of the powder particles - inherited from the droplet size distribution during the atomising process - is generally broad and only a fraction of the powder that is produced is suitable for any given additive powder-based manufacturing process. Nozzles are typically arranged in one of two configurations: close-coupled and free-fall (Figure 1). Free-fall nozzles are simpler to operate because they do not expose the melt nozzle to thermal shock and avoid problems caused by recirculation zones. Such zones can form in the close-coupled case and lead to the transport of metal droplets upwards towards the nozzle through which the melt flows, as well as the transport of fine solid particles up near the melt nozzle so that they interact with large liquid droplets. The first of these phenomena can block the melt nozzle or disrupt the flow, leading to powder particles that are outside the desired size range. The second phenomenon leads to satellites - small particles stuck on the surface of larger ones (Figure 2). Satellites are associated with a decrease in flow and may lead to a decrease in the quality of spread layers, although this latest point is still under investigation $[7,12]$. 


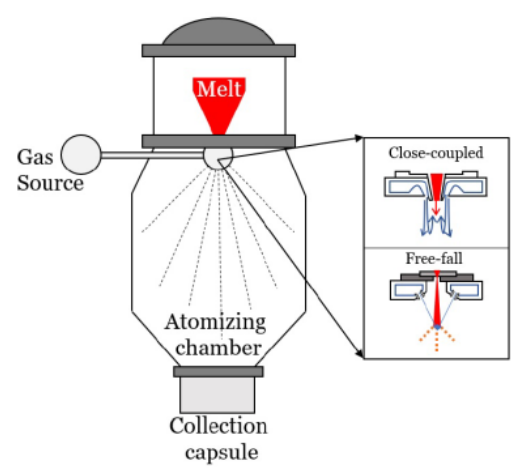

Figure 1. Simplified schematic of a gas atomiser, detailing both free-fall and close-coupled nozzle arrangements. Primary breakup occurs as soon as the gas hits the metal.

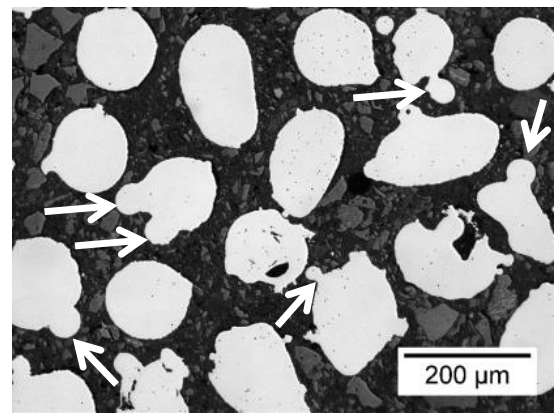

Figure 2: Optical micrograph of metal powder particles, including examples of probable satellites (arrows). The image contains more probable satellites than are marked, but only some are shown for clarity.

Traditional development of gas atomisation has been performed using a trial-andimprovement approach, with empirical analysis. Many papers have been published to predict, for example, the size of particles produced based on a range of input parameters, such as [13]. Other studies have sought to change the behaviour of the atomiser by altering the geometry, e.g. [14]. More recently, computational fluid dynamics (CFD) has emerged as a useful tool in the improvement of gas atomisation. Several distinct physical processes occur during atomisation of metal (i.e. supersonic gas flow from a nozzle, primary liquid breakup via formation of sheets and/or ligaments, secondary droplet breakup, cooling and solidification) and each of these steps must be simulated successfully to develop an acceptable and robust simulation. Many studies are underway around the world to make progress in this endeavour. Some attempt to simulate and validate the entire process, or at least several of the stages listed, in a single operation and others do not validate the numerical results at all, for example, those summarised in [13]. While these simulations may turn out to be correct, it is necessary to validate and prove each of the constituent parts so that the community may have as much confidence as possible in the results, as in [15]. This study attempts to do this for the gas flow from a single nozzle, using conditions typical of gas atomisation. This will provide a first step towards the assembly of a complex simulation for the entire gas atomiser. The same development philosophy can be used in the future to the rest of the process.

\section{Methods}

Many commercial gas atomisers use de Laval nozzles for the gas. This enables a supersonic gas flow to be achieved for high, but manageable supply pressures. The size of the de Laval nozzle is selected to achieve a desired ratio of the flow rates of gas and liquid metal. In this study, a commercially available nozzle for a free-fall atomiser has been used in both the CFD simulations and validation experiments (Figure 3). For confidentiality reasons, the precise geometry of the nozzle is not reported. 


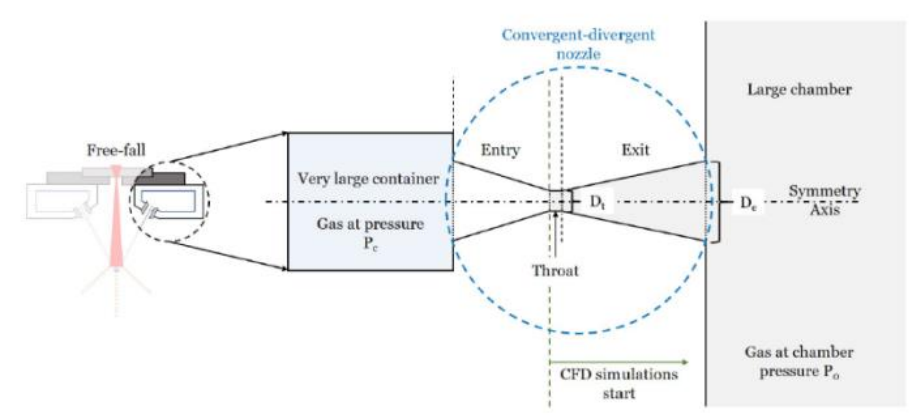

Figure 3: Schematic of the regions simulated in the CFD model and the relevance of the simulation to the gas atomising system. The "large chamber" represents the chamber where the liquid metal is atomised.

To reduce computational expense, the isentropic flow equations were used to derive the gas flow properties at the throat section of the nozzle. In this way, the upstream section of the gas flow need not be simulated. The domain of all CFD simulations in this study included the throat and diverging section of the nozzle and a large chamber after the outlet (Figure 4).

Simulations were performed in ANSYS Fluent 2019R2 using the finite volume method with an implicit, second order upwind solver and the Roe flux-difference splitting method. Turbulence was modelled using the realisable $k-\varepsilon$ equation with enhanced wall functions [16]. This was preferred to large eddy simulation (LES), despite the fact that LES is believed to produce more accurate results, especially for turbulent eddies. This was because LES demands far greater computational resources, which were not practicable in the current study, and because the eddies in the flow are not the focus of the study, rather shockwaves are, so the advantages of LES are diminished but the high cost remains. The geometry beginning of the throat was defined as a pressure inlet, which was set to an elevated pressure $\sim 20$ bar. A pressure outlet boundary was established downstream of the nozzle outlet. The pressure was set to a pressure around 1 bar. The central axis of the domain was set to be an axisymmetric boundary and the remaining surfaces were defined as walls. Fluid properties were taken directly from the values included in the materials database of ANSYS Fluent 2019R2. The turbulent intensity, $I$, at the inlet was calculated from the Reynolds number, Re, of the liquid and was found to be 0.01 (Equation 1, [17]). The same turbulent intensity persisted to the outlet.

$$
I=0.16 \mathrm{Re}^{-0.125}
$$

Simulations were run in the steady-state condition and iterated until the relative mass loss was lower than $10^{-6}$. The mesh was refined to achieve a Courant number of 2 . The mesh was finer at the nozzle exit and nozzle walls and became gradually coarser further from these features with a growth factor of 1.2. The total number of mesh elements was 695466 . The mesh density in the throat of the nozzle was $79 \mathrm{~mm}^{-2}$; at the exit and in the region where shockwaves formed, the mesh density was $249 \mathrm{~mm}^{-2}$.

Following CFD simulations, the velocity, pressure and temperature of the gas was analysed and used to calculate the refractive index, $n$, that was expected at each point of the domain. A shadowgraph shows the gradient of the refractive index of the gas (Equation 2, where $x$ is the position in the direction along the axis of symmetry, $k$ is the Gladstone-Dale coefficient and has a value of $0.23 \mathrm{~cm}^{3} \mathrm{~g}^{-1}$ for air under normal conditions [18] and $\rho$ is the gas density). The refractive index is proportional to the density of a given gas and so the shadowgraph 
effectively shows a map of the density gradient. This may be calculated within ANSYS Fluent and show a prediction of the appearance of a shadowgraph that would be made by the gas.

$$
\frac{\mathrm{d} n}{\mathrm{~d} x}=k \frac{\mathrm{d} \rho}{\mathrm{d} x}
$$

To validate the CFD results, the flow pattern of the gas after it left the nozzle was photographed from a shadowgraph and then compared to the CFD results. In shadowgraphy, parallel light is shone on the gas and changes in refractive index lead to changes in intensity of the light. This allows the flow pattern to be analysed experimentally. Parallel light was generated using a high-intensity blue LED and a fibre optic waveguide. The light was shone on a parabolic mirror to generate the parallel light. The gas nozzle was placed in this parallel light, which was incident on a second mirror to collect it and focus it to a point. This could be recorded directly using a camera or viewed on a screen placed one focal distance beyond the focal point. Images were captured using a Canon PowerShot G7 X Mark II SLR camera.

Schlieren imaging is a similar technique that adds a knife-edge at the focal point after the second mirror. This improves contrast but reduces the total illumination. Initial experiments showed that shadowgraphy gave better images than Schlieren imaging in the current set-up.

After a few seconds of flow, the shadowgraph was observed to be stable and was photographed using the camera. The photograph was converted to an 8-bit grayscale image using the image analysis program ImageJ [19]. Brightness and contrast were optimised to show the flow patterns clearly. Regions with no flow patterns were cropped out of the image.

To allow the image to be recorded, the experiment was performed in a lab that could be made completely dark. However, the only suitable lab was in a basement and did not have sufficient ventilation to allow the use of inert (asphyxiant) gases. Therefore, trials were conducted using synthetic compressed air (Linde AB, Solna, Sweden). For this reason, regular air was used as the gas in CFD simulations.

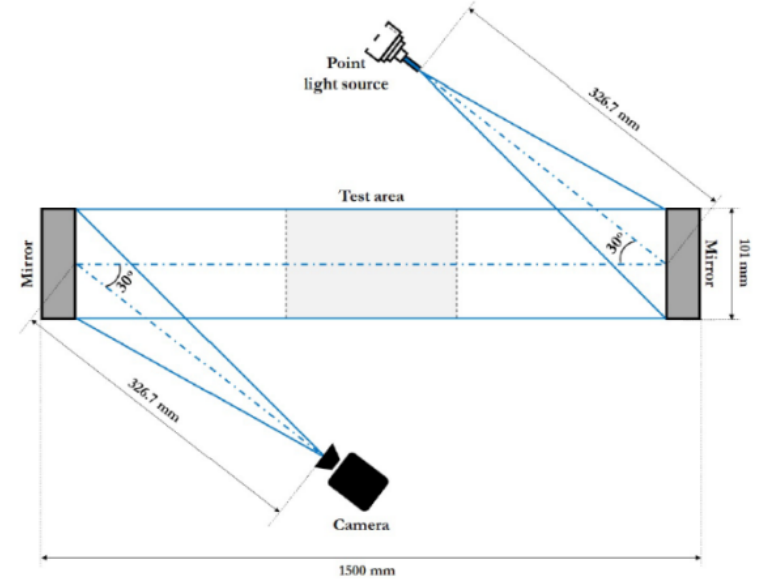

Figure 4: Schematic of the shadowgraphy setup. The gas nozzle was placed in the "test area". The mirrors were off-axis parabolic mirrors with a focal length of 326.7 " $\mathrm{mm}$ " .

\section{Results and Discussion}

The results of CFD simulations show that the gas will become supersonic and this, in turn, results in a series of shockwaves that lead to the characteristic flow pattern of gas from a de Laval nozzle (Figure 5). 
The overall design of the atomiser will dictate the ideal supply pressure, as, within the range of the pressures included in this study, large distances between the nozzle exit and the site of gas-metal interaction will lead to lower velocities for higher supply pressures. For example, at $7.5 \mathrm{~cm}$ from the nozzle exit, the gas highest velocity is seen for 20 bar supply pressure and the lowest velocity is seen for 50 bar.

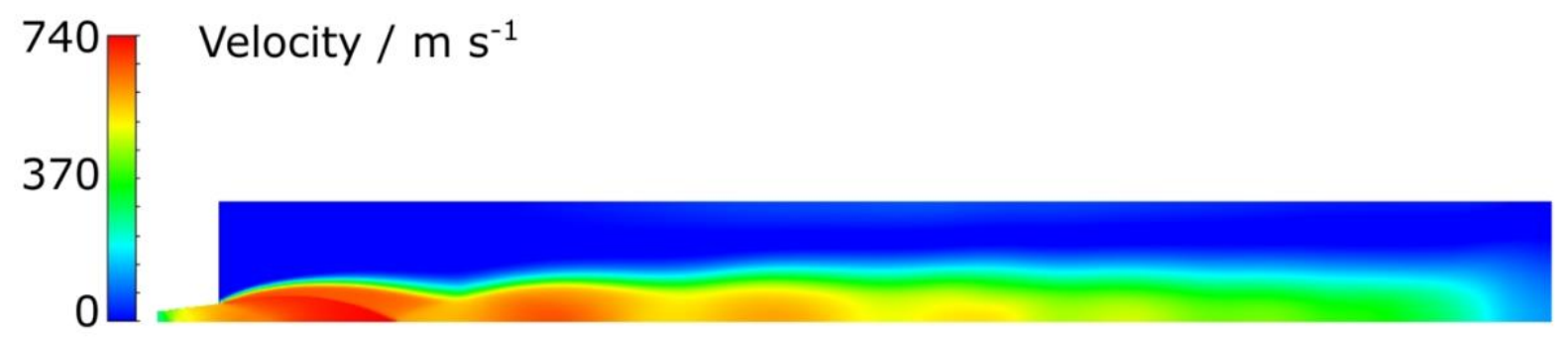

Figure 5: CFD simulation results for the velocity of the gas jet from the nozzle used in experiments with 40 bar supply pressure. The outlet pressure was set to 1" bar" , the bottom of each region is the axis of symmetry and the simulation begins on the left-hand e

The form of the calculated shadowgraph is similar to the experimentally measured image for a supply pressure of 40 bar and a chamber pressure of 1 bar, which corresponds to atmospheric pressure used in the experiment (Figure 6). The overall form of the data are similar and the number of shockwave diamonds is six in both the simulations and the experiment. However, there are two discrepancies between experiments and simulations. The first is that the length scale of the shockwave diamonds is smaller in the experiment than the simulation ( $7 \mathrm{~mm}$ cf. $22 \mathrm{~mm}$ between the second and third minima in intensity). The second is that the flow patterns were difficult to observe for the cases of 20 bar and 30 bar.

The length scale problem could simply be a function of the image being taken at the wrong point in the focal path, such that the image was demagnetised. However, the diameter of the visible region was assumed to be the diameter of the mirrors, which is a known distance and this was used to calibrate the length scale. There could also be an unknown change in pressure in the experimental system - the pressure was regulated to the nominal supply pressure (e.g. 40 bar) at the exit of a supply cylinder and could have decreased in the line before entering the nozzle. Similarly, the temperature of the gas is likely to be low due to the rapid expansion that it has undergone. Measurements using a thermocouple at the nozzle exit indicates that it is approximately $253 \mathrm{~K}$. This temperature was used in the CFD simulations to avoid this problem.

These problems may be solved by redesigning the gas system to add a second regulator immediately before the nozzle to ensure the correct supply pressure. A flowmeter could also be added to verify the volumetric flow rate in the CFD data. More temperature measurements should be taken to ensure correct temperatures are used in the CFD calculations.

The lack of observable flow pattern in the shadowgraph in the cases of 20 bar and 30 bar supply pressures may be due to low contrast. The use of air as both the background medium and the fluid being studied will lead to much smaller variations in density than would be the case if a gas such as argon or helium were used. The quality is similar to that achieved under similar conditions in a comparable study [15]. However, safety precautions prevent the use of such gases in the current set-up. During the experiments, the flow pattern was very clear in both the 20 bar and 30 bar cases, but it proved difficult to record them using the camera. Future experiments will test different cameras and/or may be performed in a different location to enable the use gases of different densities. 
(a)

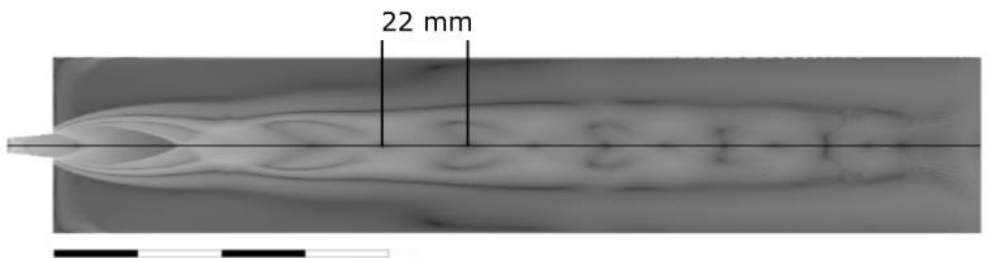

$7 \mathrm{~mm}$

(b)

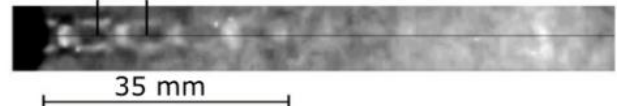

(c)

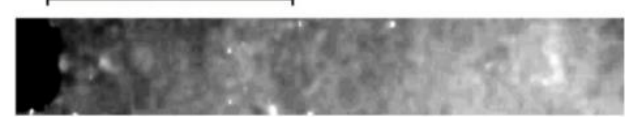

(d)

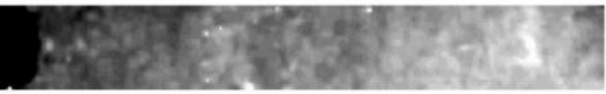

Figure 6: (a) calculated pressure gradient (high values shown as bright regions) and (b) measured shadowgraph for an inlet pressure of 40 bar (c) 30 bar and (d) 20 bar.

\section{Conclusions}

CFD calculations have been performed to calculate the expected gas flow from a de Laval nozzle under conditions that are typical for gas atomisation of liquid metals. The results show that the gas travels faster than the speed of sound in such situations and exhibits associated shock structure.

Validation of the CFD calculations using shadowgraphy has shown that the form of the flow pattern seems to be correct, but there are discrepancies between the calculations and experiments that must be investigated further.

Once the calculations have been validated successfully, more complex calculations can be performed to derive the conditions to which the liquid metal is expected to be subjected. This will allow a robust and validated model for atomisation of liquid metal to be produced, which will inform the production of metal powders for many processes, including additive manufacturing.

\section{Acknowledgments}

Chris Hulme-Smith and Arun Kamalasekaran gratefully acknowledge the support of Erasteel Kloster AB during this project.

\section{Nomenclature}

I turbulent intensity

Re Reynolds number

$n \quad$ refractive index

$x \quad$ position [m]

$\rho$ density $\left[\mathrm{g} \mathrm{cm}^{-3}\right]$

\section{References}

[1] Le Brun, P., Gaffet, E., Froyen, L., and Delaey, L., 1992, Scr. Metall. Mater., 26(11), pp. 1743-1748.

[2] Antony, L. V. M., and Reddy, R. G., 2003, JOM, 55(3), pp. 14-18.

[3] Gu, D. D., Meiners, W., Wissenbach, K., and Poprawe, R., 2012, Int. Mater. Rev., 57(3), pp. 133-164. 
[4] German, R. M., 2005, Powder Metallurgy \& Particulate Materials Processing.

[5] Yule, A. J., and Dunkley, J. J., 1994, Atomization of Melts for Powder Production and Spray Deposition.

[6] Körner, C., 2016, Int. Mater. Rev., 61(5), pp. 361-377.

[7] Cordova, L., Bor, T., de Smit, M., Campos, M., and Tinga, T., 2020, Addit. Manuf., 32(January), p. 101082.

[8] Spierings, A. B., Herres, N., and Levy, G., 2011, Rapid Prototyp. J., 17(3), pp. 195202.

[9] Shrestha, S., and Manogharan, G., 2017, JOM, 69(3), pp. 491-497.

[10] Cordova, L., Campos, M., and Tinga, T., 2013, VI Congreso Nacional de Pulvimetalurgia y I Congreso Iberoamericano de Pulvimetalurgia.

[11] International Standards Organisation, 2020, SS-EN ISO 52907:2020 Additive Manufacturing - Feedstock Materials - Methods to Characterize Metal Powders, ISO.

[12] Mussatto, A., Groarke, R., O'Neill, A., Obeidi, M. A., Delaure, Y., and Brabazon, D., 2021, Addit. Manuf., 38(November), p. 101807.

[13] Sarkar, S., Sivaprasad, P. V., and Bakshi, S., 2016, At. Sprays, 26(1), pp. 23-51.

[14] Fritsching, U., and Uhlenwinke, V., 2012, Powder Metall., pp. 99-124.

[15] Motaman, S., Mullis, A. M., Cochrane, R. F., and Borman, D. J., 2015, Metall. Mater. Trans. B Process Metall. Mater. Process. Sci., 46(4), pp. 1990-2004.

[16] Versteeg, H., and Malalasekera, W., 2007, An Introduction to Computational Fluid Dynamics: The Finite Volume Method.

[17] ANSYS, 2009, https://www.afs.enea.it/project/neptunius/docs/fluent/html/ug/main_pre.htm.

[18] Settles, G. S., 2001, Schlieren and Shadowgraph Techniques.

[19] Schneider, C. A., Rasband, W. S., and Eliceiri, K. W., 2012, Nat. Methods, 9(7), pp. 671-675. 